\title{
Scalable self-growth of Ni@NiO core-shell electrode with ultrahigh capacitance and super-long cyclic stability for supercapacitors
}

\author{
Minghao Yu, Wang Wang, Cheng Li, Teng Zhai, Xihong Lu and Yexiang Tong
}

Three-dimensional (3D) electrodes have been demonstrated to be promising candidates for high-performance supercapacitors because of their unique architectures and outstanding electrochemical properties. However, the fabrication process for current 3D electrodes is not scalable. Herein, a novel and cost-effective activation process has been developed to macroscopically produce 3D porous $\mathrm{Ni@NiO}$ core-shell electrodes with enhanced electrochemical properties. The porous $\mathrm{Ni@NiO}$ core-shell electrode obtained by activated commercial $\mathrm{Ni}$ foam (NF) in a $3 \mathrm{M} \mathrm{HCl}$ solution yields an ultrahigh areal capacitance of $2.0 \mathrm{~F} \mathrm{~cm}^{-2}$ at a high current density of $8 \mathrm{~mA} \mathrm{~cm}{ }^{-2}$, which is substantially higher than that of most reported 3D NF-based electrodes. Moreover, the activated NF (ANF) electrode exhibited super-long cycling stability. Owing to the increased accessible surface area and continual formation of electrochemically active NiO during cycling, the areal capacitance of the ANF electrode did not exhibit any decay and instead increased from 0.47 to $1.27 \mathrm{~F} \mathrm{~cm}^{-2}$ after 100000 cycles at $100 \mathrm{mV} \mathrm{s}^{-1}$. This is the best cycling stability achieved by a 3D NF-based electrode. Additionally, a high-performance asymmetrical supercapacitor (ASC) device based on the as-prepared ANF cathode and a reduced graphene oxide (RGO) anode was also prepared. The ANF//RGOASC device was able to deliver a maximum energy density of $1.06 \mathrm{mWh} \mathrm{cm}{ }^{-3}$ and a maximum power density of $0.42 \mathrm{~W} \mathrm{~cm}$. NPG Asia Materials (2014) 6, e129; doi:10.1038/am.2014.78; published online 19 September 2014

\section{INTRODUCTION}

The ever-increasing demands for renewable energy and growing concerns of environmental protection have stimulated extensive interest in the development of efficient energy conversion and storage devices. ${ }^{1-4}$ Supercapacitors (SCs), also known as electrochemical capacitors, are widely recognized as promising candidates for energy storage devices because these devices have high power density, excellent stability (up to 100000 cycles) and low maintenance costs. ${ }^{1,2,4-7}$ These features make SCs favorable for applications in high-power electronic devices, emergency power supplies and hybrid electric vehicles. However, the energy density of SCs is relatively low, which severely limits their practical applications as primary power sources. Considerable efforts have been devoted to increasing the energy density of SCs, and great advances have been made recently. One promising strategy to improve the energy density is to design nanostructured electrode materials with large surface areas and good structural stability. Various nanostructures including nanoparticles, ${ }^{8}$ nanowires, ${ }^{9,10}$ nanotubes ${ }^{11}$ and nanosheets ${ }^{12,13}$ have been explored as SC electrodes and have exhibited improved electrochemical performance. Among these nanostructures, three-dimensional (3D) porous nanoarchitectures have been demonstrated to be a promising candidate for SCs as these nanoarchitectures can offer large interfacial areas, reduced ionic diffusion distances and facilitate charge separation and transport. For instance, 3D graphene-based electrodes, such as graphene/carbon nanotubes, ${ }^{14}$ graphene/ $\mathrm{Co}_{3} \mathrm{O}_{4}{ }^{15}$ and graphene/ $\mathrm{MnO}_{2}, 16,17$ have been developed. Despite these achievements, it is highly desirable to develop a simple and scalable method for the construction of high-performance 3D electrodes.

Nickel foam (NF), a porous structure with an interconnected 3D scaffold of Ni metal, possesses unique features such as exceptional uniformity, lightweightness, high porosity (50-95\%), intrinsic strength, corrosion resistance and good electrical and thermal conductivity. Thus, NF has drawn significant attention as a support (current collector) and/or template for constructing 3D electrodes. Numerous 3D electrodes with excellent electrochemical performance, such as $\mathrm{NiCO}_{2} \mathrm{O}_{4},{ }^{18} \mathrm{MnO}_{2},{ }^{16} \mathrm{Ni}(\mathrm{OH})_{2},{ }^{19}$ graphene ${ }^{20,21}$ and $\mathrm{Co}_{3} \mathrm{O}_{4},{ }^{22}$ have been developed through the direct growth of these materials on NF using a hydrothermal method, electrodeposition and chemical vapor deposition. However, the fabrication process for these electrodes requires the deposition of active materials into the deep pores of NF, which limits the loading amount of active materials and often yields inhomogeneous structures with diminished capacitance. Moreover, the complex and difficult-to-scale process severely reduces their practical applications, especially for large-scale energy storage

KLGHEI of Environment and Energy Chemistry, MOE of the Key Laboratory of Bioinorganic and Synthetic Chemistry, School of Chemistry and Chemical Engineering, Sun Yat-Sen University, Guangzhou, People's Republic of China

Correspondence: Dr X Lu or Professor Y Tong, KLGHEl of Environment and Energy Chemistry, MOE of the Key Laboratory of Bioinorganic and Synthetic, School of Chemistry and Chemical Engineering, Sun Yat-Sen University, 135 Xingang West Road, Chemical North Building 325, Guangzhou 510275, People's Republic of China.

E-mail: luxh6@mail.sysu.edu.cn or chedhx@mail.sysu.edu.cn

Received 12 May 2014; revised 8 July 2014; accepted 28 July 2014 
applications. The search for simple approaches to massively produce high-performance 3D electrodes has been highly pursued.

In this work, we report the facile and large-scale synthesis of highperformance 3D Ni@NiO core-shell electrodes by activating commercial NF using a one-step and cost-effective approach under mild conditions. A porous and 3D Ni@NiO core-shell electrode with excellent electrochemical property has been prepared by simply immersing $\mathrm{NF}$ into a $3 \mathrm{M} \mathrm{HCl}$ aqueous solution at $90{ }^{\circ} \mathrm{C}$ for $20 \mathrm{~min}$. Electrochemical measurements confirmed that the activated $\mathrm{Ni}$ foam (ANF) electrode achieved an ultrahigh areal capacitance of $2.0 \mathrm{~F} \mathrm{~cm}^{-2}$ at a high current density of $8 \mathrm{~mA} \mathrm{~cm}^{-2}$, which offers an $\sim 10$-fold improvement over a bare NF electrode. Moreover, this ANF electrode possessed an extraordinary cyclic stability without any capacitance decay and an $\sim 170 \%$ enhancement in capacitance after 100000 cycles. In addition, in comparison with other NF-based 3D electrodes reported recently, this present activation process has the advantages of simplicity, low cost and scalable production that can yield a large output with an area of $600 \mathrm{~cm}^{2}$ or larger in one activation process. This high performance of ANF also offers new opportunities in the design and fabrication of high-performance SCs. An asymmetric SC (ASC) device using the as-prepared ANF as the cathode and reduced graphene oxide (RGO) as the anode achieved a maximum volumetric energy density of $1.06 \mathrm{mWh} \mathrm{cm}^{-3}$ and a maximum volumetric power density of $0.42 \mathrm{Wcm}^{-3}$, which are substantially higher than the values obtained for most reported SCs. ${ }^{1,9,16,23-29}$

\section{MATERIALS AND METHODS}

\section{Activation of NF}

All reagents used were of analytical grade and were used directly without any purification. Before activation, an entire piece of NF $(29.7 \mathrm{~cm} \times 21 \mathrm{~cm} \times 0.1$ $\mathrm{cm}$, mass per unit area of $25.5 \mathrm{mg} \mathrm{cm}^{-2}$, from Changsha Lyrun New Material, Changsha, People's Republic of China) was cleaned with alcohol, acetone and distilled water in turn. Typically, the entire piece of NF was directly immersed into 21 of a $3 \mathrm{M} \mathrm{HCl}$ aqueous solution, followed by $20 \mathrm{~min}$ of static soaking in the lab environment at $90^{\circ} \mathrm{C}$. Then, the NF was removed and cleaned with distilled water to remove any extra $\mathrm{HCl}$ solution. Finally, the NF was dried at room temperature.

\section{Fabrication of RGO electrodes}

Fabrication of RGO electrodes followed the method described in our recent work. $^{20}$ Briefly, a graphene oxide suspension was first prepared by the oxidation of natural graphite powder using the modified Hummers' method. Then, the NF $(3 \mathrm{~cm} \times 4 \mathrm{~cm})$ was immersed in $40 \mathrm{ml}$ of the graphene suspension $\left(2 \mathrm{mg} \mathrm{ml}^{-1}\right)$ in a $50-\mathrm{ml}$ Teflon-lined stainless-steel autoclave. The sealed autoclave was heated at $180^{\circ} \mathrm{C}$ for $3 \mathrm{~h}$. After the autoclave was naturally cooled to room temperature, the as-prepared RGO electrode was repeatedly rinsed with deionized water and dried. To prepare RGO electrodes with sufficient areal capacitance, the above process was repeated on one piece of $\mathrm{NF}$ six times.

\section{Fabrication of aqueous ASC}

The aqueous ASC device was assembled using an ANF electrode $(0.5 \mathrm{~cm} \times 3 \mathrm{~cm})$ and RGO electrode $(0.5 \mathrm{~cm} \times 3 \mathrm{~cm})$ with a separator (NKK separator; Nippon Kodoshi Corporation, Kochi, Japan) sandwiched between these electrodes. A $\mathrm{KOH}(6 \mathrm{M})$ aqueous solution was used as the electrolyte. To avoid leakage of the electrolyte, the entire device was sealed in a small plastic bag with a small part of electrode kept outside.

\section{Materials characterization and electrochemical measurements}

The microstructures and compositions of the electrode materials were analyzed using scanning electron microscopy (SEM; JSM-6330F, JOEL, Tokyo, Japan), Raman spectroscopy (inVia, Renishaw plc, Gloucestershire, UK), X-ray photoelectron spectroscopy (XPS, ESCALab250; Thermo VG, Beverly, MA, USA) and X-ray diffractometry (D8 ADVANCE, Bruker Corporation, Saarbrücken, Germany). The surface area of the product was calculated from nitrogen adsorption/desorption isotherms at $77 \mathrm{~K}$ that were obtained using an ASAP 2020 V3.03 H instrument (Micromeritics, Norcross, GA, USA). Before measurement, all the samples were outgassed at $100^{\circ} \mathrm{C}$ for $300 \mathrm{~min}$ under flowing nitrogen. Cyclic voltammetry (CV), galvanostatic charge/discharge measurements and electrochemical impedance spectroscopy were conducted using an electrochemical workstation (CHI 760E, Shanghai Chen hua instrument co., LTD, Shanghai, China). The electrochemical studies of the individual electrode were performed in a conventional three-electrode cell, with a Pt counter electrode and a saturated calomel reference electrode.

\section{RESULTS AND DISCUSSION}

The 3D ANF was obtained using a one-step facile and scalable activation process, which is schematically illustrated in Figure 1a. Briefly, a piece of cleaned NF, with a size of A4 paper, was directly immersed into $3 \mathrm{M} \mathrm{HCl}$ aqueous solution and maintained at $90^{\circ} \mathrm{C}$ for $20 \mathrm{~min}$ (see Materials and methods). During the activation process, the inert nickel oxide arising from the oxidation of the surface nickel in air and the surface $\mathrm{Ni}$ were first dissolved in the $\mathrm{HCl}$ solution, which is confirmed by the changing color of the $\mathrm{HCl}$ solution (Supplementary Figure S1). Then, the newly exposed nickel with high activity reacted immediately with the oxygen dissolved in the solution or in the air to form new nickel oxide. The color of the NF film turned from white to black after this activation (the insets in Figures $1 \mathrm{~b}$ and $\mathrm{c}$ ), indicating the formation of nickel oxide. SEM images confirmed that the smooth surface of the bare NF became rough and numerous ravines were formed on the NF electrode during the activation process (Figures $1 \mathrm{~b}$ and $\mathrm{c}$ ).

To determine the possible phase and composition changes during the activation process, we performed X-ray diffraction, Raman and XPS analyses on the NF and ANF. The X-ray diffractometry studies suggest that the main composition of NF and ANF is nickel, as no other peaks were detected for either the NF and ANF samples (Supplementary Figure S2). Figure 2a presents the typical Raman spectra of NF and ANF. No characteristic Raman scattering was observed for the pristine NF, which indicates the metallic nature and relatively high purity of the NF. In contrast, the ANF sample exhibits two sharp peaks at 448 and $519 \mathrm{~cm}^{-1}$, which are well assigned to the first-order transverse optical and longitudinal optical phonon modes of NiO. ${ }^{21,30}$ This result reveals that fresh $\mathrm{NiO}$ was formed on the surface of NF after activation, which is further confirmed by the XPS analyses. Figure $2 \mathrm{~b}$ presents XPS survey spectra of the NF and ANF samples. The $\mathrm{C}$ signal was derived from the adventitious carbon. Only $\mathrm{Ni}$ and $\mathrm{O}$ signals are detected for both the NF and ANF samples, indicating that the activation process did not introduce other impurities into NF. Figure 2c compares the core level Ni 2p XPS spectra of the NF and ANF samples. Six clear peaks are observed for both samples. The peaks centered at 852.4 and $869.5 \mathrm{eV}$ correspond to the characteristic $\mathrm{Ni} 2 \mathrm{p}_{3 / 2}$ and $\mathrm{Ni} 2 \mathrm{p}_{1 / 2}$ peaks of $\mathrm{Ni}^{0}$, respectively, whereas the peaks located at 856.1 and $873.8 \mathrm{eV}$ represent the characteristic $\mathrm{Ni} 2 \mathrm{p}_{3 / 2}$ and $\mathrm{Ni} 2 \mathrm{p}_{1 / 2}$ peaks of $\mathrm{Ni}^{2+}$, respectively. ${ }^{31,32}$ In comparison with the pristine NF sample, the intensities of the characteristic $\mathrm{Ni}^{2+}$ peaks of the ANF sample are much higher, indicating that the NF surface was oxidized after activation. Additionally, the substantially lower intensity of the characteristic $\mathrm{Ni}^{0}$ peaks also confirms the formation of nickel oxide on the ANF surface. Figure $2 \mathrm{~d}$ presents the core level O 1s XPS spectra of the NF and ANF samples, which can be deconvoluted into two peaks. The peaks at $531.1 \mathrm{eV}$ are attributed to $\mathrm{Ni}-\mathrm{O}-\mathrm{Ni}$, whereas the peaks at $532.1 \mathrm{eV}$ are attributed to $\mathrm{Ni}-\mathrm{O}-\mathrm{H}$ at the surface of the $\mathrm{NF}$ and 
a
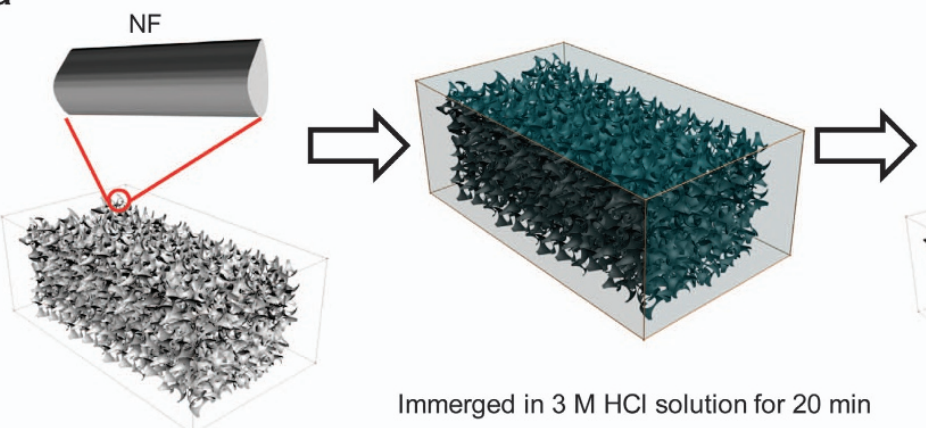

Immerged in $3 \mathrm{M} \mathrm{HCl}$ solution for $20 \mathrm{~min}$

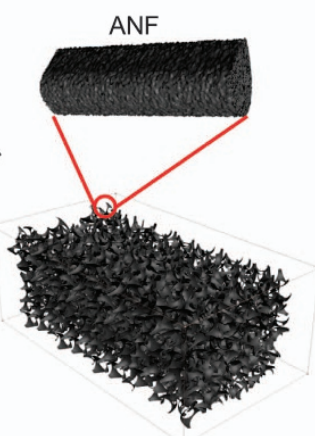

b

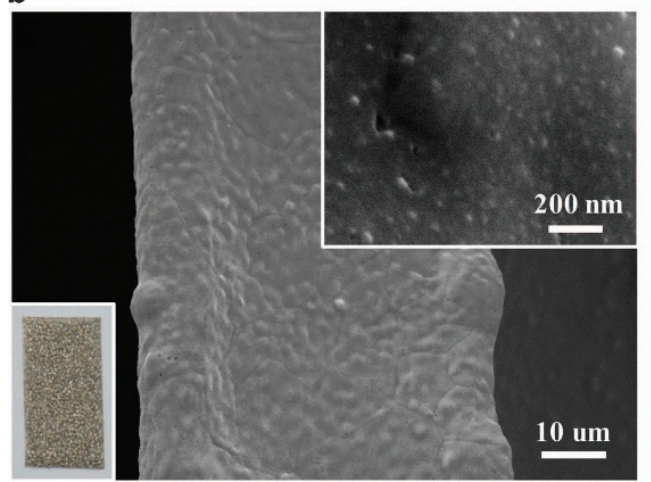

C

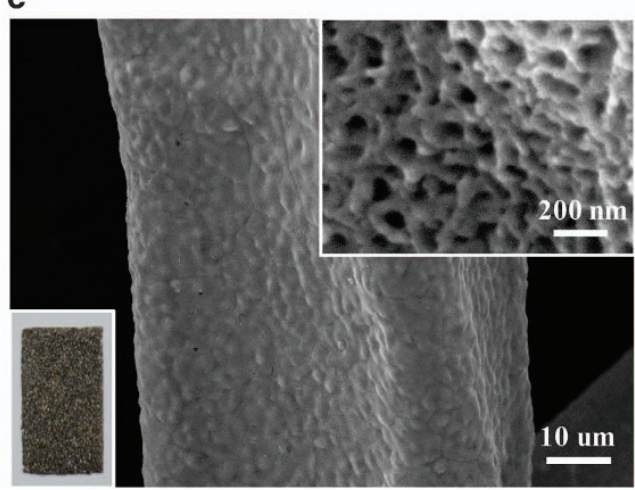

Figure 1 (a) Schematic diagram illustrating the activation process of the Ni foam (NF). (b) Scanning electron microscopy (SEM) images of bare NF. (c) SEM images of the as-prepared activated NF (ANF). The insets of (b) and (c) are the corresponding magnified SEM images and digital photos.

ANF. $^{33,34}$ The Ni-O peak intensity of the ANF sample is substantially higher than that of the NF sample, again confirming that a layer of $\mathrm{NiO}$ was formed on the NF surface after immersion into the $\mathrm{HCl}$ aqueous solution at $90^{\circ} \mathrm{C}$ for $20 \mathrm{~min}$. All these results fully validate that ANF consists of a $\mathrm{Ni}$ core and an enriched $\mathrm{NiO}$ shell.

To evaluate the electrochemical performance of the ANF electrode, electrochemical measurements were conducted in a three-electrode cell using $6 \mathrm{~m} \mathrm{KOH}$ aqueous solution as the electrolyte with a Pt wire counter electrode and a saturated calomel electrode reference electrode. Figure $3 \mathrm{a}$ compares the $\mathrm{CV}$ curves of the $\mathrm{NF}$ and $\mathrm{ANF}$ electrodes collected at a scan rate of $100 \mathrm{mV} \mathrm{s}^{-1}$. Significantly, the ANF electrode exhibited a substantially larger current density than the $\mathrm{NF}$ electrode, indicating the superior capacitive performance of the ANF electrode. CV curves of the ANF electrode collected at different scan rates are presented in Figure 3b. A pair of clear redox peaks that correspond to the reversible Faradic redox reaction, $\mathrm{NiO}+\mathrm{OH}^{-} \leftrightarrow$ $\mathrm{NiOOH}+e^{-}$, are observed in each curve, illustrating the typical pseudocapacitive behavior of the ANF electrode. Additionally, the good symmetrical characteristics of the anodic and cathodic peaks indicate the redox reversibility of the ANF electrode. Because it was difficult to measure the mass of electrochemically active $\mathrm{NiO}$ in both the NF and ANF electrodes, we calculated the areal capacitances to replace the specific capacitance. As shown in Supplementary Figure $\mathrm{S} 3 \mathrm{~b}$, the ANF electrode exhibits much higher areal capacitances than the NF electrode and yields the highest areal capacitance of $1.43 \mathrm{~F} \mathrm{~cm}^{-2}$ at the scan rate of $5 \mathrm{mV} \mathrm{s}^{-1}$. Moreover, the ANF electrode has a good rate capability with more than $33 \%$ retention of capacitance as the scan rate increases from 5 to $100 \mathrm{mV} \mathrm{s}^{-1}$. This outstanding electrochemical performance of the ANF are attributed to the increased surface area (the specific surface area calculated using the Brunauer-Emmett-Teller method is approximately $5.9 \mathrm{~m}^{2} \mathrm{~g}^{-1}$ for the ANF, which is increased by more than twofold over the NF $\left.\left(2.2 \mathrm{~m}^{2} \mathrm{~g}^{-1}\right)\right)$ and the enriched electrochemically active $\mathrm{NiO}$ after activation.

The superior electrochemical performance of the ANF electrode was further confirmed by galvanostatic charge/discharge measurements. Figure $3 \mathrm{c}$ presents the galvanostatic charge/discharge curves of the NF and ANF electrodes. Owing to the low electrical double layer capacitance, a rapid charging and discharging process was observed at $0-0.2$ and $0.4-0.2 \mathrm{~V}$. The much longer discharge time of the ANF electrode demonstrates its preferable electrochemical performance to that of the NF electrode. The areal capacitance of the ANF electrode reached $2.04 \mathrm{~F} \mathrm{~cm}^{-2}$ at the current density of $8 \mathrm{~mA} \mathrm{~cm}^{-2}$ (Figure 3d), which is more than 10 times that of the value obtained for the NF electrode $\left(0.20 \mathrm{~F} \mathrm{~cm}^{-2}\right)$. This present areal capacitance obtained at such a high discharge current density is also considerably higher than that of recently reported NF-based electrodes, such as $\mathrm{Ni}(\mathrm{OH})_{2} @ N F$ $\left(1.6 \mathrm{~F} \mathrm{~cm}^{-2}\right.$ at $\left.2 \mathrm{mAcm}^{-2}\right), 35 \quad \mathrm{NiCo}_{2} \mathrm{O}_{4} @ \mathrm{NF} \quad\left(1.5 \mathrm{~F} \mathrm{~cm}^{-2}\right.$ at $\left.5 \mathrm{mAcm}^{-2}\right),{ }^{36} \quad \mathrm{CoMoO}_{4} @ \mathrm{NF} \quad\left(1.26 \mathrm{~F} \mathrm{~cm}^{-2}\right.$ at $\left.4 \mathrm{~mA} \mathrm{~cm}^{-2}\right),{ }^{37}$ CoO@NF $\left(1.23 \mathrm{~F} \mathrm{~cm}^{-2} \text { at } 1 \mathrm{~mA} \mathrm{~cm}{ }^{-2}\right)^{23}$ and $\mathrm{MnO}_{2} @ \mathrm{NF}\left(0.25 \mathrm{~F} \mathrm{~cm}^{-2}\right.$ at $\left.1.03 \mathrm{mAcm}^{-2}\right) .{ }^{38}$ Moreover, the ANF electrode exhibits an excellent rate capability with more than $55 \%$ retention of the initial capacitance as the current density increases from 8 to $20 \mathrm{~mA} \mathrm{~cm}^{-2}$. This remarkable capacitance and rate capability of the ANF electrode can be attributed to its unique structural features: (1) a 3D Ni core with high conductivity enables efficient charge transport and accessible diffusion of the electrolyte; (2) the direct connection between the electrochemically active $\mathrm{NiO}$ shell and $\mathrm{Ni}$ core can effectively facilitate the interfacial charge transfer; and (3) the rough surface and porous architecture of the ANF electrode not only significantly increase the accessible surface area as well as active sites for redox reaction but also promote the fast intercalation and deintercalation of ions. 
a

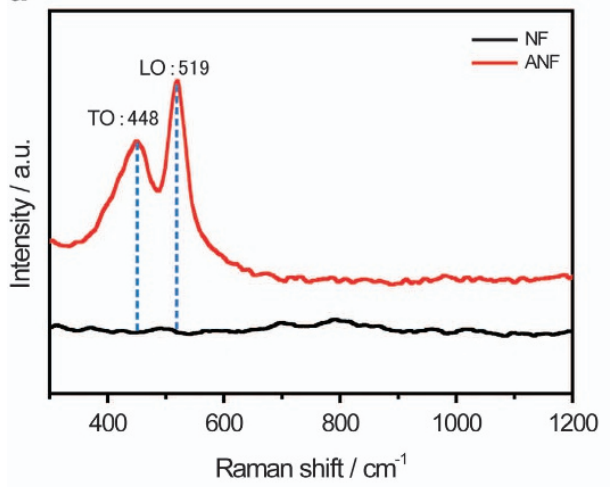

c

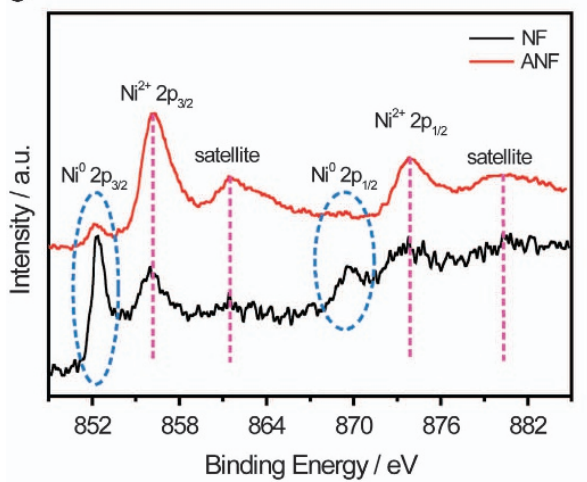

b

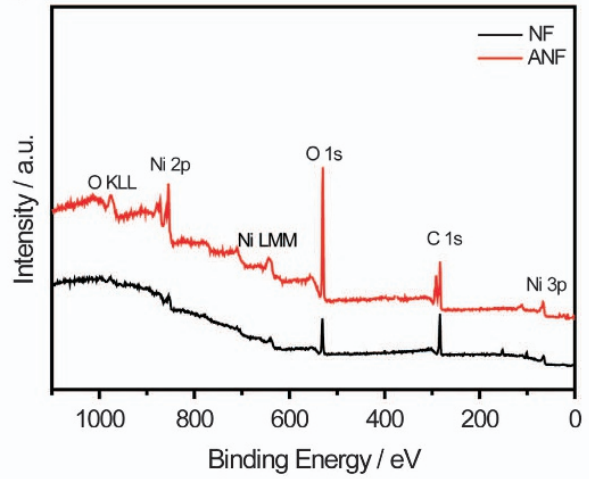

d

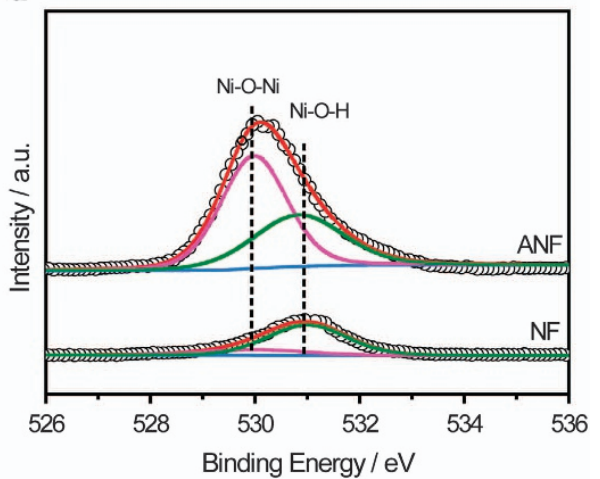

Figure 2 (a) Raman spectra, (b) survey X-ray photoelectron spectroscopy (XPS) spectra, (c) Ni 2p XPS spectra and (d) 0 1s XPS spectra of Ni foam (NF) and activated NF (ANF).

We also studied the long-term cycling performance of the ANF electrode. Figure 4a shows the variation of the areal capacitance collected for the ANF electrode at a scan rate of $100 \mathrm{mV} \mathrm{s}^{-1}$ for 100000 cycles. Notably, the areal capacitance of the ANF electrode continuously increased in the initial 30000 cycles and then remained comparatively steady as the cycling continued. After 100000 cycles, the ANF electrode achieves $1.27 \mathrm{~F} \mathrm{~cm}^{2}$, which represents an $\sim 170 \%$ enhancement compared with its initial capacitance before cycling $\left(0.47 \mathrm{~F} \mathrm{~cm}^{-2}\right)$. To the best of our knowledge, such cyclic stability has not been reported for 3D electrodes to date. The electrochemical performance of the ANF electrode after 30000 cycles was further investigated, and good capacitive behavior was maintained (Supplementary Figure S4). Figure 4b compares the CV curves for ANF before and after 30000 cycles collected at $100 \mathrm{mV} \mathrm{s}^{-1}$. In addition to the increased current density, the intensity of the redox peaks also obviously increased, which may be attributed to the further enrichment of $\mathrm{NiO}$ during the cycling test. To understand the origin of the enhanced capacitance of the ANF electrode, the morphology and chemical composition of the ANF electrode after 30000 cycles were investigated. SEM images reveal that the surface of the ANF electrode became rougher and porous compared with the surface before cycling (Figures $1 \mathrm{c}$ and $4 \mathrm{c}$ ), indicating that the surface area of the ANF electrode was greatly increased after 30000 cycles. Figure $4 \mathrm{~d}$ compares the core level Ni 2p XPS spectra of the ANF electrode before and after 30000 cycles. The intensities of the $\mathrm{Ni}^{2+}$ peaks markedly increase, and the $\mathrm{Ni}^{0}$ peaks almost disappear after 30000 cycles. This result indicates that the surface of the ANF electrode was continually oxidized and activated during the charging and discharging process. The same conclusion was drawn based on the Raman analysis
(Figure 4e). More and much stronger characteristic Raman peaks of $\mathrm{NiO}$ are observed for the ANF electrode after cycling, again confirming that electrochemically active $\mathrm{NiO}$ was continually formed on the ANF surface with increasing cycles. Therefore, the enhanced performance of the ANF electrode during the cycling test is because of the increased accessible surface area (a substantially enhanced specific surface area of approximately $8.3 \mathrm{~m}^{2} \mathrm{~g}^{-1}$ was attained, which was calculated using Brunauer-Emmett-Teller method) and continual formation of electrochemically active $\mathrm{NiO}$ (from the oxidization of newly exposed $\mathrm{Ni}$ during both the activation and cycling test processes). Additionally, the morphology and chemical composition remained stable, which was confirmed by the comparison of the SEM images and XPS spectra for the ANF electrode after 30000 and 100000 cycles (Supplementary Figure S5). This finding indicates that the electrochemical performance of the ANF electrode can be improved through this activation process.

To demonstrate the feasibility of the ANF as a high-performance electrode in SCs, we assembled an ASC using the ANF after the 30000 -cycle $\mathrm{CV}$ test at $100 \mathrm{mV}^{-1}$ as the cathode and RGO as the anode. The RGO was coated on NF as described in the literature (Supplementary Information). ${ }^{16}$ SEM images and CV curves of the RGO electrode are presented in Supplementary Figure S6. Before the assembly, the charge between the ANF and RGO electrodes needed to be balanced, and the areal ratio of the RGO to ANF after the 30000 cycle CV test at $100 \mathrm{mV}^{-1}$ was calculated to be $1.07: 1$ (for details, see the Supplementary Information and Supplementary Figure S7). Figure 5a presents the CV curves of the optimized ANF//RGO-ASC device collected at $50 \mathrm{mVs}^{-1}$ with different voltage windows, demonstrating that the stable voltage window of the as-fabricated 
a

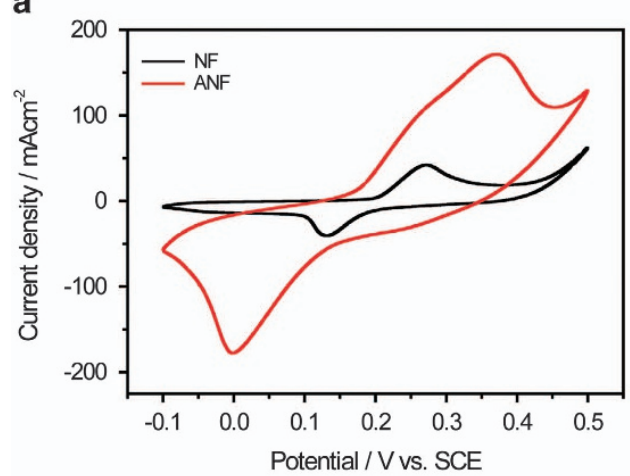

C

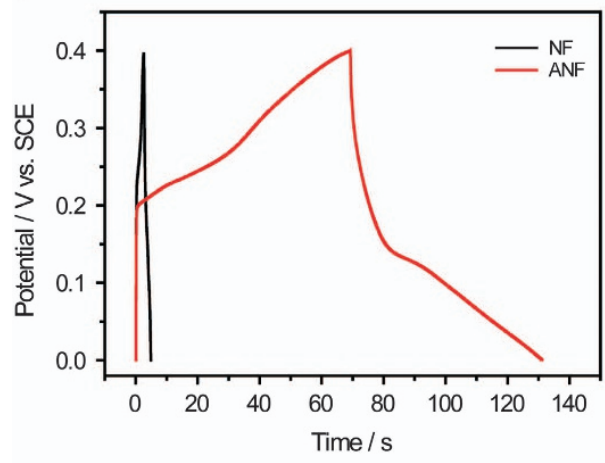

b

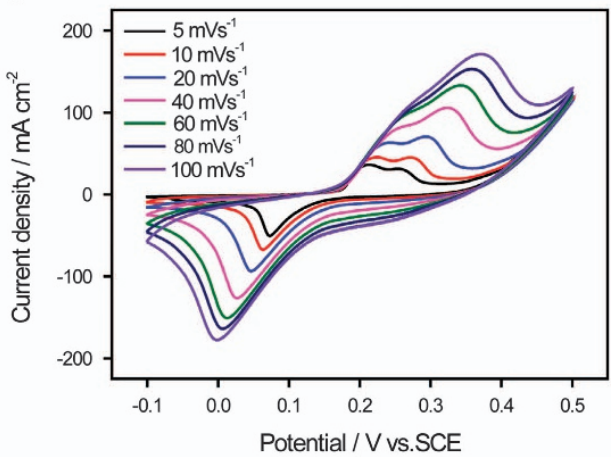

d

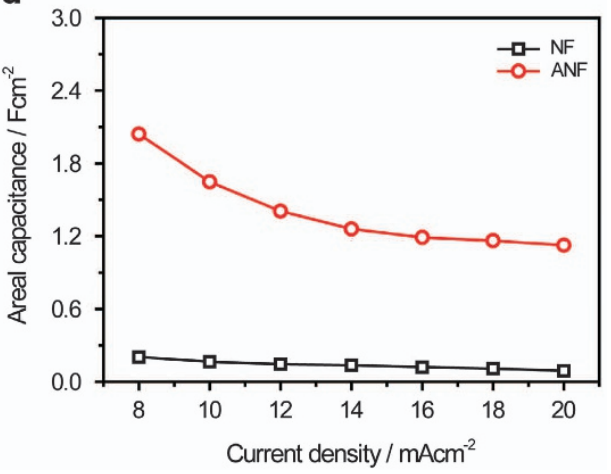

Figure 3 (a) Cyclic voltammetry (CV) curves collected at $100 \mathrm{mVs}^{-1}$ of the $\mathrm{Ni}$ foam (NF) and activated NF (ANF) electrodes. (b) CV curves of the ANF electrode collected at various scan rates. (c) Galvanostatic charge/discharge curves of the NF and ANF electrodes. (d) Areal capacitance as a function of the current density of the NF and ANF electrodes.

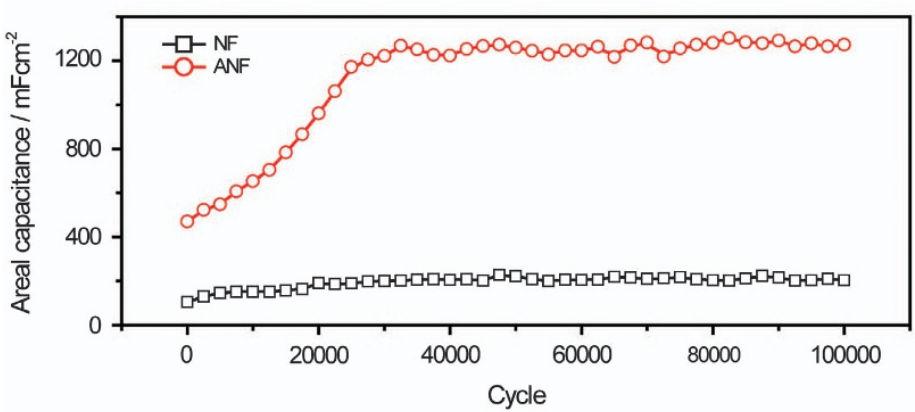

C

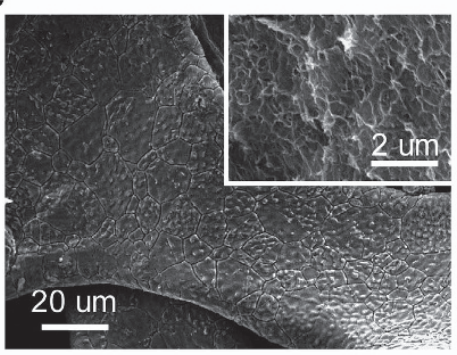

b

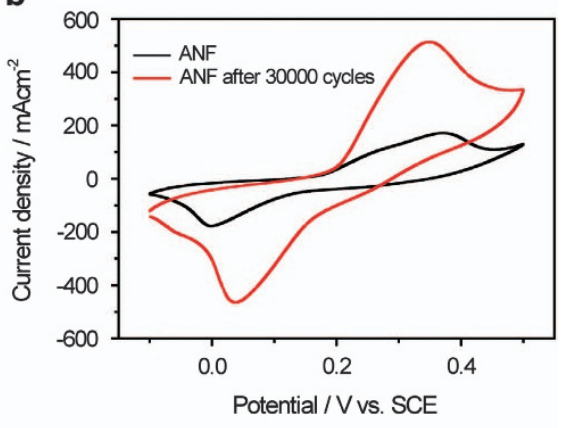

e

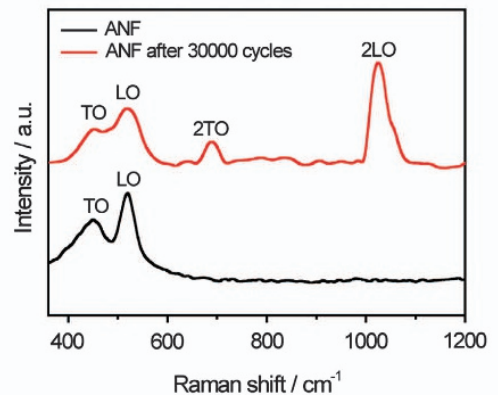

Figure 4 (a) Cycling performance of the Ni foam (NF) and activated NF (ANF) electrodes collected at $100 \mathrm{mVs}^{-1}$. (b) Cyclic voltammetry (CV) curves collected at $100 \mathrm{mVs}^{-1}$ of the ANF electrode and ANF electrode after 30000 cycles. (c) Scanning electron microscopy (SEM) images of the ANF electrode after 30000 cycles. (d) Ni 2p X-ray photoelectron spectroscopy (XPS) and (e) Raman spectra of ANF before and after 30000 cycles. 
a

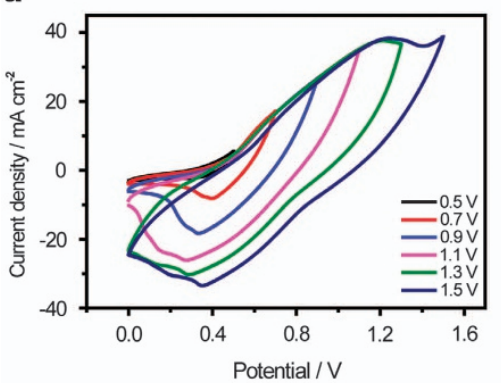

d

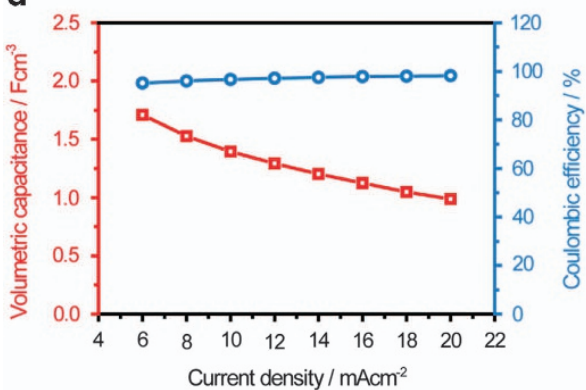

b

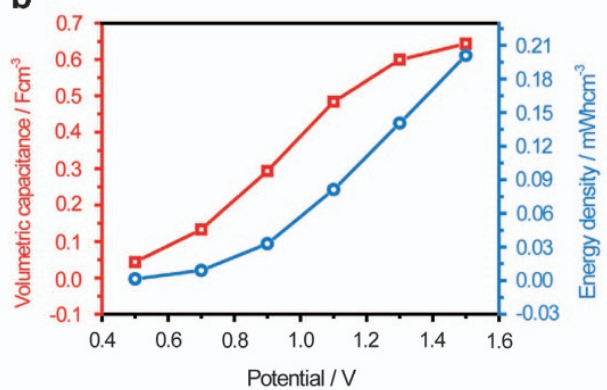

e

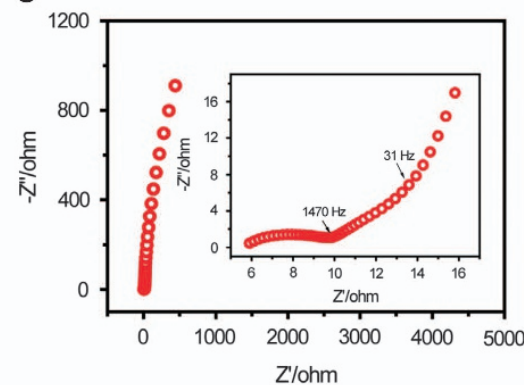

C

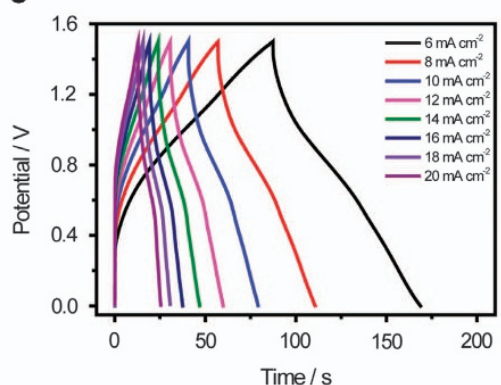

f

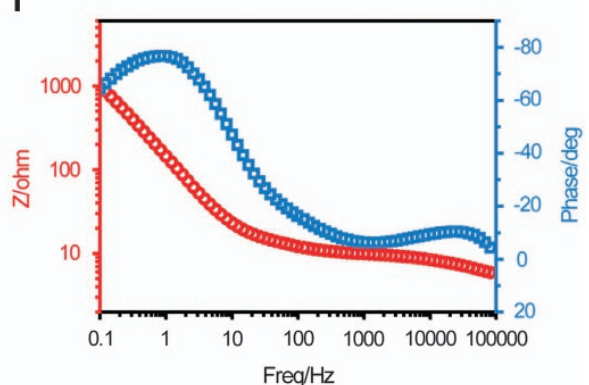

Figure 5 (a) Cyclic voltammetry (CV) curves of the activated NF (ANF)//reduced graphene oxide-asymmetrical supercapacitor (RGO-ASC) device collected for different scan voltage windows at $50 \mathrm{mVs}^{-1}$. (b) Volumetric capacitance and energy density calculated for the ASC device as a function of potential windows. (c) Galvanostatic charge/discharge curves collected at different current densities for the ASC device operated within a voltage window of $1.5 \mathrm{~V}$. (d) Volumetric capacitance and coulombic efficiency for the ANF//RGO-ASC device as a function of current density. (e) Nyquist and (f) Bode plots of the ANF//RGO-ASC device.

ASC device can be extended to $1.5 \mathrm{~V}$ without damage of the cycling stability (Supplementary Figure S8). Additionally, the volumetric capacitance of this ASC device (based on the volume of the entire device) was significantly increased (from 0.08 to $1.38 \mathrm{~F} \mathrm{~cm}^{-3}$ ) as the operation voltage increased from 0.6 to $1.5 \mathrm{~V}$ (Figure $5 \mathrm{~b}$ ). In addition, the energy density of the ASC device was also markedlyly improved

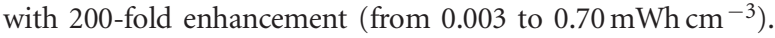

Figure $5 \mathrm{c}$ displays the galvanostatic charge/discharge curves of the ANF//RGO-ASC device collected at different current densities. The relatively symmetric shapes of these curves indicate the ideal capacitive characteristics and rapid charge/discharge properties of the ANF//RGO-ASC device. As shown in Figure 5d, the ANF//RGOASC device achieved the highest volumetric capacitance of $3.42 \mathrm{~F} \mathrm{~cm}^{-3}$ at $6 \mathrm{mAcm}^{-2}$, which is substantially higher than the values reported recently for other ASCs, such as an NiO//RGO-ASC device $\left(1.37 \mathrm{~F} \mathrm{~cm}^{-3}\right.$ at $\left.1 \mathrm{mAcm}^{-2}\right),{ }^{39}$ an $\mathrm{MnO}_{2} / \mathrm{RGO} / / \mathrm{RGO}-\mathrm{ASC}$ device $\left(1.60 \mathrm{~F} \mathrm{~cm}^{-3}\right.$ at $\left.4 \mathrm{mAcm}^{-2}\right),{ }^{16}$ a $\mathrm{VO}_{x} / / \mathrm{VN}-\mathrm{ASC}$ device $\left(1.35 \mathrm{~F} \mathrm{~cm}^{-3}\right.$ at $\left.0.5 \mathrm{~mA} \mathrm{~cm}^{-2}\right),{ }^{9}$ a $\mathrm{TiO}_{2} @ \mathrm{MnO}_{2} / / \mathrm{TiO}_{2} @ \mathrm{C}-\mathrm{ASC}$ device $\left(0.67 \mathrm{~F} \mathrm{~cm}^{-3} \text { at } 0.5 \mathrm{~mA} \mathrm{~cm}^{-2}\right)^{28}$ and a $\mathrm{ZnO@MnO} / / \mathrm{RGO}-$ ASC device $\left(0.52 \mathrm{~F} \mathrm{~cm}^{-3}\right.$ at $\left.0.5 \mathrm{mAcm}^{-2}\right) .{ }^{24}$ In addition, when the current density increased from 6 to $20 \mathrm{~mA} \mathrm{~cm}^{-2}$, the ANF//RGO-ASC device retained $58 \%$ of its capacitance, demonstrating its good rate capability, and more than $95 \%$ of the coulombic efficiency was retained at any current density.

To further evaluate the electrochemical behaviors of the asfabricated ASC device, electrochemical impedance spectroscopy analysis was performed. Nyquist plots of the as-fabricated ASC device are presented in Figure 5e. The straight line parallel to the imaginary axis reveals the ideal capacitive behavior of the ANF//RGO-ASC device. A small charge-transfer resistance of $\sim 4 \Omega$ was observed for the ANF//RGO-ASC device, indicating its fast charge transfer property. The transition between the RC semicircle and the migration

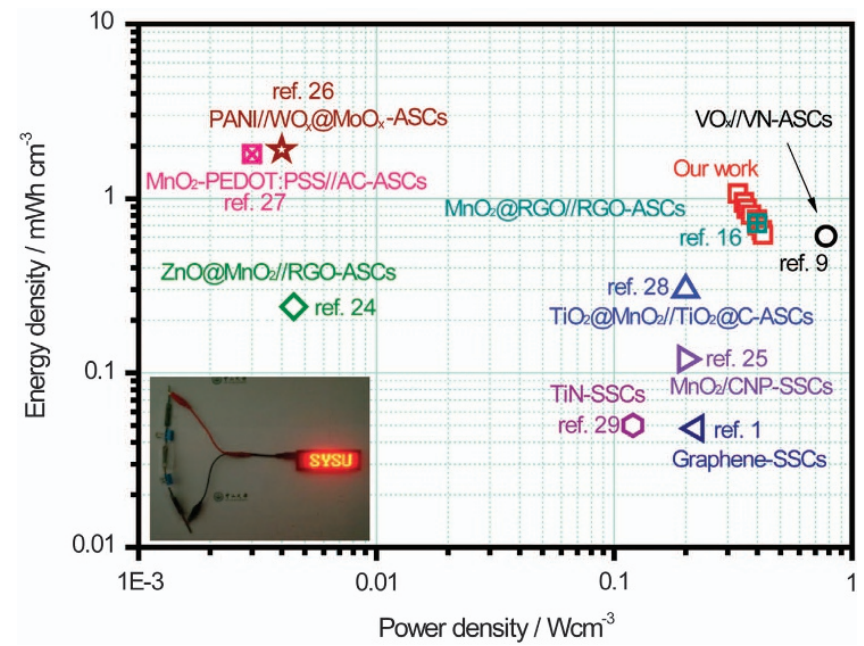

Figure 6 Ragone plots of the quasi-solid-state activated NF (ANF)//reduced graphene oxide-asymmetrical supercapacitor (RGO-ASC) device. The values reported for the other supercapacitor (SC) devices are added for comparison. CNP, carbon nanoparticle; SSC, symmetric SC; PANI, polyaniline; PEDOT, Poly(3,4-ethylenedioxythiophene); PSS, polystyrene sulfonate. $1,9,16,24-29$

of the electrolyte was observed at $1470 \mathrm{~Hz}$, and the ASCs display a pure capacitive behavior even at a high frequency up to $31 \mathrm{~Hz}$. Figure $5 \mathrm{f}$ presents Bode plots of the ANF//RGO-ASC device. The phase angle of the ASC device reached $-77^{\circ}$ at $1 \mathrm{~Hz}$, indicating that the functionality of the as-fabricated ASC device is close to that of an ideal capacitor. The characteristic frequency $f_{0}$ for a phase angle of $-45^{\circ}$ is $\sim 11 \mathrm{~Hz}$ (Figure $5 \mathrm{f}$ ). According to the equation $\tau_{0}=1 / f_{0}$, the relaxation time constant $\tau_{0}$ of this ASC device is calculated to be 
$\sim 0.09 \mathrm{~s}$, which is substantially shorter than that of recently reported SCs. ${ }^{9,25}$ Such a rapid frequency response further confirms the fast ion transport rate property of the ANF//RGO-ASC device. Additionally, the ANF//RGO-ASC device exhibits outstanding cycling stability without any decay volumetric capacitance after 10000 cycles (Supplementary Figure S8).

As two important parameters for evaluating the electrochemical performance of an SC device, we have calculated the energy and power densities of the ANF//RGO-ASC device and compared these results with other developed SC devices (Figure 6, detailed calculation, see Supplementary Information). Significantly, although our asfabricated ANF//RGO-ASC device only has a small voltage window of $1.5 \mathrm{~V}$, the maximum volumetric energy density reached $1.06 \mathrm{mWh} \mathrm{cm}^{-3}$. This value is substantially higher than that of recently reported symmetric SCs (SSCs) and most of ASCs with larger operation potential windows, such as graphene-SSC $\left(0.07 \mathrm{mWh} \mathrm{cm}^{-3}\right),{ }^{1} \mathrm{MnO}_{2} / \mathrm{CNP}-\mathrm{SSC}\left(0.12 \mathrm{mWh} \mathrm{cm}^{-3}\right),{ }^{25} \mathrm{TiN}-\mathrm{SSC}$ $\left(0.05 \mathrm{mWh} \mathrm{cm}^{-3}\right),{ }^{29} \quad \mathrm{ZnO} @ \mathrm{MnO}_{2} / / \mathrm{RGO}-\mathrm{ASC} \quad\left(0.24 \mathrm{mWh} \mathrm{cm}^{-3}\right.$, $1.8 \mathrm{~V})^{24}$ and $\mathrm{TiO}_{2} @ \mathrm{MnO}_{2} / / \mathrm{TiO}_{2} @ \mathrm{C}-\mathrm{ASC}\left(0.30 \mathrm{mWh} \mathrm{cm}^{-3}, 1.8 \mathrm{~V}\right){ }^{28}$ are comparable with that of $\mathrm{VO}_{x} / / \mathrm{VN}-\mathrm{ASC}\left(0.61 \mathrm{mWh} \mathrm{cm}^{-3}, 1.8 \mathrm{~V}\right)^{9}$ and $\mathrm{MnO}_{2} / \mathrm{RGO} / / \mathrm{RGO}-\mathrm{ASC}\left(0.72 \mathrm{mWh} \mathrm{cm}^{-3}, 1.8 \mathrm{~V}\right) .{ }^{16}$ Moreover, the ANF//RGO-ASC device also exhibited a superior power density of $0.42 \mathrm{~W} \mathrm{~cm}^{-3}$, which is considerably higher than that of SSCs and ASCs. ${ }^{1,23-29}$ After charging at a current density of $20 \mathrm{~mA} \mathrm{~cm}^{-2}$ for $10 \mathrm{~s}$, a tandem device with three ANF//RGO-ASC devices in series could power a light-emitting diode array display $(4-5 \mathrm{~V})$ for $\sim 1 \mathrm{~min}$ (inset in Figure 5). Moreover, because of the unique porous structure and strong mechanical properties, the volumetric performance of the ANF//RGO-ASC device can be further improved by compression of the device. For instance, the volumetric capacitance of the compressed ANF//RGO-ASC device reached $4.99 \mathrm{~F} \mathrm{~cm}^{-3}$ at $6 \mathrm{mAcm}^{-2}$, resulting in a maximum energy density of $1.56 \mathrm{mWh} \mathrm{cm}^{-3}$ (Supplementary Figures S10 and S11). All of these findings convincingly demonstrate that the ANF is a high-performance electrode for ASCs.

\section{CONCLUSIONS}

In summary, a one-step effective activation method has been developed to produce 3D Ni@NiO core-shell electrodes on a large scale with enhanced electrochemical properties for SCs. After being immersed into a $3 \mathrm{M} \mathrm{HCl}$ aqueous solution, the 3D NF became rough and porous and formed an electrochemically active $\mathrm{NiO}$ shell on its surface. This pore-enriched nanostructure provides abundant open channels for ion transport and a highly accessible interface for redox reactions, and the $\mathrm{Ni@NiO}$ core-shell structure facilitates charge collection and transport. Benefiting from this unique architecture, the ANF electrode yields a remarkable areal capacitance of $2.0 \mathrm{~F} \mathrm{~cm}^{-2}$ at a high current density of $8 \mathrm{mAcm}^{-2}$, which is approximately 10 times that of the unactivated NF electrode. Moreover, the ANF electrode exhibited ultrahigh long-term cycling stability without any decay of capacitance after 100000 cycles. Additionally, a highperformance ASC device based on the ANF cathode and RGO anode was also prepared. The ANF//RGO-ASC device achieved a maximum energy density of $1.06 \mathrm{mWh} \mathrm{cm}^{-3}$ and a maximum power density of $0.42 \mathrm{~W} \mathrm{~cm}^{-3}$. Significantly, the ANF//RGO-ASC device exhibits excellent cycling stability without any decay of volumetric capacitance after 10000 cycles. This work constitutes the first demonstration of directly using ANF as a high-energy electrode, which could potentially improve the performance of energy storage devices.

\section{CONFLICT OF INTEREST}

The authors declare no conflict of interest.

\section{ACKNOWLEDGEMENTS}

YXT acknowledges the financial support of this work received by the Natural Science Foundation of China (21273290, 91323101 and J1103305) and the Research Fund for the Doctoral Program of Higher Education of China (20120171110043). XHL acknowledges the Young Teacher Starting-up Research of Sun Yat-sen University. WW acknowledges the National Undergraduates Innovating Experimentation Project.

1 El-Kady, M. F., Strong, V., Dubin, S., Kaner, R. B. Laser scribing of high-performance and flexible graphene-based electrochemical capacitors. Science 335, 1326-1330 (2012).

2 Zhu, Y., Murali, S., Stoller, M. D., Ganesh, K. J., Cai, W., Ferreira, P. J., Pirkle, A., Wallace, R. M., Cychosz, K. A., Thommes, M., Su, D., Stach, E. A., Ruoff, R. S. Carbon-based supercapacitors produced by activation of graphene. Science 332, 1537-1541 (2011).

3 Luo, J., Liu, J., Zeng, Z., Ng, C. F., Ma, L., Zhang, H., Lin, J., Shen, Z., Fan, H. J. Three-dimensional graphene foam supported $\mathrm{Fe}(3) \mathrm{O}(4)$ lithium battery anodes with long cycle life and high rate capability. Nano Lett. 13, 6136-6143 (2013).

4 Xia, X., Zhu, C., Luo, J., Zeng, Z., Guan, C., Ng, C. F., Zhang, H., Fan, H. J. Synthesis of free-standing metal sulfide nanoarrays via anion exchange reaction and their electrochemical energy storage application. Small 10, 766-773 (2014).

5 Lu, X., Zeng, Y., Yu, M., Zhai, T., Liang, C., Xie, S., Balogun, M. S., Tong, Y. Oxygen-deficient hematite nanorods as high-performance and novel negative electrodes for flexible asymmetric supercapacitors. Adv. Mater. 26, 3148-3155 (2014).

6 Guan, C., Zeng, Z., Li, X., Cao, X., Fan, Y., Xia, X., Pan, G., Zhang, H., Fan, H. J. Atomic-layer-deposition-assisted formation of carbon nanoflakes on metal oxides and energy storage application. Small 10, 300-307 (2014).

$7 \mathrm{Yu}, \mathrm{Z}$., Thomas, J. Energy storing electrical cables: integrating energy storage and electrical conduction. Adv. Mater. 26, 4279-4285 (2014).

8 Bastakoti, B. P., Oveisi, H., Hu, C.-C., Wu, K. C.-W., Suzuki, N., Takai, K., Kamachi, Y., Imura, M., Yamauchi, Y. Mesoporous carbon incorporated with $\ln _{2} \mathrm{O}_{3}$ nanoparticles as high-performance supercapacitors. Eur. J. Inorg. Chem. 2013, 1109-1112 (2013).

9 Lu, X., Yu, M., Zhai, T., Wang, G., Xie, S., Liu, T., Liang, C., Tong, Y., Li, Y. High energy density asymmetric quasi-solid-state supercapacitor based on porous vanadium nitride nanowire anode. Nano Lett. 13, 2628-2633 (2013).

10 Lu, X., Liu, T., Zhai, T., Wang, G., Yu, M., Xie, S., Ling, Y., Liang, C., Tong, Y., Li, Y. Improving the cycling stability of metal-nitride supercapacitor electrodes with a thin carbon shell. Adv. Energy Mater. 4, 1300994 (2014).

11 Niu, Z., Luan, P., Shao, Q., Dong, H., Li, J., Chen, J., Zhao, D., Cai, L., Zhou, W., Chen, X., Xie, S. A "skeleton/skin" strategy for preparing ultrathin free-standing singlewalled carbon nanotube/polyaniline films for high performance supercapacitor electrodes. Energy Environ. Sci. 5, 8726-8733 (2012).

12 Zhou, W., Cao, X., Zeng, Z., Shi, W., Zhu, Y., Yan, Q., Liu, H., Wang, J., Zhang, H. Onestep synthesis of $\mathrm{Ni}_{3} \mathrm{~S}_{2}$ nanorod@Ni(OH)$)_{2}$ nanosheet core-shell nanostructures on a three-dimensional graphene network for high-performance supercapacitors. Energy Environ. Sci. 6, 2216-2221 (2013).

13 Wu, C., Lu, X., Peng, L., Xu, K., Peng, X., Huang, J., Yu, G., Xie, Y. Two-dimensional vanadyl phosphate ultrathin nanosheets for high energy density and flexible pseudocapacitors. Nat. Commun. 4, 2431 (2013).

14 Zhu, Y., Li, L., Zhang, C., Casillas, G., Sun, Z., Yan, Z., Ruan, G., Peng, Z., Raji, A. R., Kittrell, C., Hauge, R. H., Tour, J. M. A seamless three-dimensional carbon nanotube graphene hybrid material. Nat. Commun 3, 1225 (2012).

15 Dong, X. C., Xu, H., Wang, X. W., Huang, Y. X., Chan-Park, M. B., Zhang, H., Wang, L. H., Huang, W., Chen, P. 3D graphene-cobalt oxide electrode for high-performance supercapacitor and enzymeless glucose detection. ACS Nano 6, 3206-3213 (2012).

16 Zhai, T., Wang, F., Yu, M., Xie, S., Liang, C., Li, C., Xiao, F., Tang, R., Wu, Q., Lu, X., Tong, Y. 3D MnO2-graphene composites with large areal capacitance for highperformance asymmetric supercapacitors. Nanoscale 5, 6790-6796 (2013).

17 Xia, X., Chao, D., Fan, Z., Guan, C., Cao, X., Zhang, H., Fan, H. J. A new type of porous graphite foams and their integrated composites with oxide/polymer core/shell nanowires for supercapacitors: structural design, fabrication, and full supercapacitor demonstrations. Nano Lett. 14, 1651-1658 (2014).

18 Zhang, G., Lou, X. W. General solution growth of mesoporous $\mathrm{NiCO}_{2} \mathrm{O}_{4}$ nanosheets on various conductive substrates as high-performance electrodes for supercapacitors. Adv. Mater. 25, 976-979 (2013).

19 Wang, H., Liang, Y., Mirfakhrai, T., Chen, Z., Casalongue, H. S., Dai, H. Advanced asymmetrical supercapacitors based on graphene hybrid materials. Nano Res. 4, 729-736 (2011).

20 Chen, Z., Ren, W., Gao, L., Liu, B., Pei, S., Cheng, H. M. Three-dimensional flexible and conductive interconnected graphene networks grown by chemical vapor deposition. Nat. Mater. 10, 424-428 (2011).

21 Cao, X., Shi, Y., Shi, W., Lu, G., Huang, X., Yan, Q., Zhang, Q., Zhang, H. Preparation of novel 3D graphene networks for supercapacitor applications. Small 7, 3163-3168 (2011).

22 Zhang, F., Yuan, C., Lu, X., Zhang, L., Che, Q., Zhang, X. Facile growth of mesoporous $\mathrm{CO}_{3} \mathrm{O}_{4}$ nanowire arrays on $\mathrm{Ni}$ foam for high performance electrochemical capacitors. J. Power Sources 203, 250-256 (2012). 
23 Zhou, C., Zhang, Y., Li, Y., Liu, J. Construction of high-capacitance 3D CoO@polypyrrole nanowire array electrode for aqueous asymmetric supercapacitor. Nano Lett. 13, 2078-2085 (2013).

24 Zilong, W., Zhu, Z., Qiu, J., Yang, S. High performance flexible solid-state asymmetric supercapacitors from $\mathrm{MnO}_{2} / \mathrm{ZnO}$ core-shell nanorods//specially reduced graphene oxide. J. Mater. Chem. C 2, 1331-1336 (2014).

25 Yuan, L., Lu, X. H., Xiao, X., Zhai, T., Dai, J., Zhang, F., Hu, B., Wang, X., Gong, L., Chen, J., Hu, C., Tong, Y., Zhou, J., Wang, Z. L. Flexible solid-state supercapacitors based on carbon nanoparticles $/ \mathrm{MnO}_{2}$ nanorods hybrid structure. ACS Nano 6 , 656-661 (2012).

26 Xiao, X., Ding, T., Yuan, L., Shen, Y., Zhong, Q., Zhang, X., Cao, Y., Hu, B., Zhai, T., Gong, L., Chen, J., Tong, Y., Zhou, J., Wang, Z. L. WO3 $-x / \mathrm{MoO}_{3}-\mathrm{xCore} /$ Shell nanowires on carbon fabric as an anode for all-solid-state asymmetric supercapacitors. Adv. Energy Mater. 2, 1328-1332 (2012).

27 Su, Z., Yang, C., Xu, C., Wu, H., Zhang, Z., Liu, T., Zhang, C., Yang, Q., Li, B., Kang, F. Co-electro-deposition of the $\mathrm{MnO}_{2}$-PEDOT:PSS nanostructured composite for high areal mass, flexible asymmetric supercapacitor devices. J. Mater. Chem. A 1, 1243212440 (2013).

28 Lu, X., Yu, M., Wang, G., Zhai, T., Xie, S., Ling, Y., Tong, Y., Li, Y. H-TiO(2) @MnO(2)// $\mathrm{H}-\mathrm{TiO}(2) @ \mathrm{C}$ core-shell nanowires for high performance and flexible asymmetric supercapacitors. Adv. Mater. 25, 267-272 (2013).

29 Lu, X., Wang, G., Zhai, T., Yu, M., Xie, S., Ling, Y., Liang, C., Tong, Y., Li, Y. Stabilized TiN nanowire arrays for high-performance and flexible supercapacitors. Nano Lett. 12, 5376-5381 (2012).

30 Wang, W. Z., Liu, Y. K., Xu, C. K., Zheng, C. L., Wang, G. H. Synthesis of NiO nanorods by a novel simple precursor thermal decomposition approach. Chem. Phys. Lett. 362, 119-122 (2002).

31 Peck, M. A., Langell, M. A. Comparison of nanoscaled and bulk NiO structural and environmental characteristics by XRD, XAFS, and XPS. Chem. Mater. 24, 4483-4490 (2012).

32 Biesinger, M. C., Lau, L. W., Gerson, A. R., Smart, R. S. The role of the Auger parameter in XPS studies of nickel metal, halides and oxides. Phys. Chem. Chem. Phys. 14, 2434-2442 (2012).
33 Kim, S. I., Lee, J. S., Ahn, H. J., Song, H. K., Jang, J. H. Facile route to an efficient $\mathrm{NiO}$ supercapacitor with a three-dimensional nanonetwork morphology. ACS Appl. Mater. Interfaces 5, 1596-1603 (2013).

34 Salvati, L., Makovsky, L. E., Stencel, J. M., Brown, F. R., Hercules, D. M. Surface spectroscopic study of tungsten-alumina catalysts using x-ray photoelectron, ion scattering, and Raman spectroscopies. J. Phys. Chem. 85, 3700-3707 (1981).

35 Yang, G. W., Xu, C. L., Li, H. L. Electrodeposited nickel hydroxide on nickel foam with ultrahigh capacitance. Chem. Commun. 6537-6539 (2008).

$36 \mathrm{Yu}$, L., Zhang, G., Yuan, C., Lou, X. W. Hierarchical $\mathrm{NiC}_{2} \mathrm{O}_{4} @ \mathrm{MnO}_{2}$ core-shell heterostructured nanowire arrays on $\mathrm{Ni}$ foam as high-performance supercapacitor electrodes. Chem. Commun. 49, 137-139 (2013)

37 Guo, D., Zhang, H., Yu, X., Zhang, M., Zhang, P., Li, Q., Wang, T. Facile synthesis and excellent electrochemical properties of $\mathrm{CoMoO}_{4}$ nanoplate arrays as supercapacitors. J. Mater. Chem. A 1, 7247-7254 (2013).

38 Yan, D., Guo, Z., Zhu, G., Yu, Z., Xu, H., Yu, A. $\mathrm{MnO}_{2}$ film with three-dimensional structure prepared by hydrothermal process for supercapacitor. J. Power Sources 199, 409-412 (2012).

39 Luan, F., Wang, G., Ling, Y., Lu, X., Wang, H., Tong, Y., Liu, X. X., Li, Y. High energy density asymmetric supercapacitors with a nickel oxide nanoflake cathode and a 3D reduced graphene oxide anode. Nanoscale 5, 7984-7990 (2013).

(c) (1) (2) This work is licensed under a Creative Commons Attribution-NonCommercial-ShareAlike 3.0 Unported License. The images or other third party material in this article are included in the article's Creative Commons license, unless indicated otherwise in the credit line; if the material is not included under the Creative Commons license, users will need to obtain permission from the license holder to reproduce the material. To view a copy of this license, visit http://creativecommons.org/licenses/by-nc-sa/3.0/

Supplementary Information accompanies the paper on the NPG Asia Materials website (http://www.nature.com/am) 